\title{
An Adaptive Geometrical Compensation Control Scheme for Hydraulic Manipulators
}

\author{
Zhou, Jianjun; Conrad, Finn; Sørensen, Paul Haase
}

Published in:

Proceedings of the IEEE International Workshop on Intelligent Motion Control

Publication date:

1990

Document Version

Publisher's PDF, also known as Version of record

Link back to DTU Orbit

Citation (APA):

Zhou, J., Conrad, F., \& Sørensen, P. H. (1990). An Adaptive Geometrical Compensation Control Scheme for Hydraulic Manipulators. In Proceedings of the IEEE International Workshop on Intelligent Motion Control (pp. 4347). IEEE.

\section{General rights}

Copyright and moral rights for the publications made accessible in the public portal are retained by the authors and/or other copyright owners and it is a condition of accessing publications that users recognise and abide by the legal requirements associated with these rights.

- Users may download and print one copy of any publication from the public portal for the purpose of private study or research.

- You may not further distribute the material or use it for any profit-making activity or commercial gain

- You may freely distribute the URL identifying the publication in the public portal

If you believe that this document breaches copyright please contact us providing details, and we will remove access to the work immediately and investigate your claim. 


\title{
AN ADAPTIVE GEOMETRICAL COMPENSATION CONTROL SCHEME FOR HYDRAULIC MANIPULATORS
}

\author{
Jian-jun Zhou1, Finn Conrad, Paul H. Sørensen \\ Control Engineering Institute \\ Building 424 \\ Technical University of Denmark \\ 2800 Lyngby Denmark
}

\begin{abstract}
An adaptive geometrical compensation control scheme is proposed for hydraulic manipulators. The scheme consists of feedforward geometric compensation, computed on the basis of the desired joint velocity, and adaptive feedback control for correcting the deviations from the desired trajectory. The simulation results confirm the effectiveness of the proposed control scheme.
\end{abstract}

\section{INTRODUCTION}

At present, most industrial robot control systems utilize independent PID joint controllers. However, the simple independent joint control is not adequate for the case when the robot manipulators move at high speed and in situations requiring a precise tracking of the trajectories and sustaining a desirable high dynamic performance for varying payloads.

To overcome the shortcoming of the independent joint control, a Iu umber of advanced control schemes have been proposed during the past decade, where most of the advanced control schemes utilizes dynamic compensation techniques, e.g. computed-torque control [1], feedforward control [2], and adaptive perturbation control [3]. The dynamic compensation in these schemes may in principle be performed either on-line or off-line. However, in the on-line calculation approach, computation time is a major problem and a heavy computational burden tends to limit the adaptation rates significantly. Furthermore, when the systems under consideration do not have a large memory capacity at the joint level, the off-line calculation method is unfeasible.

In this paper an adaptive geometric compensation scheme is proposed for hydraulic manipulators, which consists of feedforward geometric compensation, computed on the basis of the desired joint velocity, and adaptive feedback control to force the robot to track the desired trajectory. Because the scheme does not require computation of the inverse robot arm dynamics, the feedforward compensation can be easily implemented on-line.

For expository convenience, the control scheme is developed based on a two-link hydraulic test robot which was designed and constructed at the Control Engineering Institute, Technical University of Denmark [4-5]. The results can be extended to other manipulators.

\section{A TWO-LINK HYDRAULIC TEST ROBOT}

The constructed hydraulic test robot is shown in Fig. 1 . The robot arms are directly driven by hydraulic actuators which are controlled by super-high frequency response servovalves. The cylinders in the actuators are symmetric with double piston rods. The controller is implemented with an AT\&T WE-DSP32 signal processor, which has the machine cycle time of $40 \mathrm{nsec}$. One of the goals of designing and constructing the hydraulic test robot is to investigate the feasibility of implementing adaptive control techniques for tast robots.

The robot arm dynamics can be described by a Lagrange-Euler equation of motion

$$
D(q) \ddot{q}+H(q, \dot{q})+G(q)=\tau
$$

where $q, \dot{q}$, and $\ddot{q}$ are vectors signifying the joint positions, velocities, and accelerations, respectively, $D(q)$ is an acceleration-related matrix, $H(q, \dot{q})$ is a Coriolis and centrifugal force vector, $G(q)$ is a gravitational force vector, and $\tau$ is an applied torque vector generated by the actuators.

The actuator equations consist of a servovalve dynamic equat on, a cylinder continuity equation, a static valve equation, and a pis on

1 from Beijing University of Aeronautics and Astronautics, Cr.na

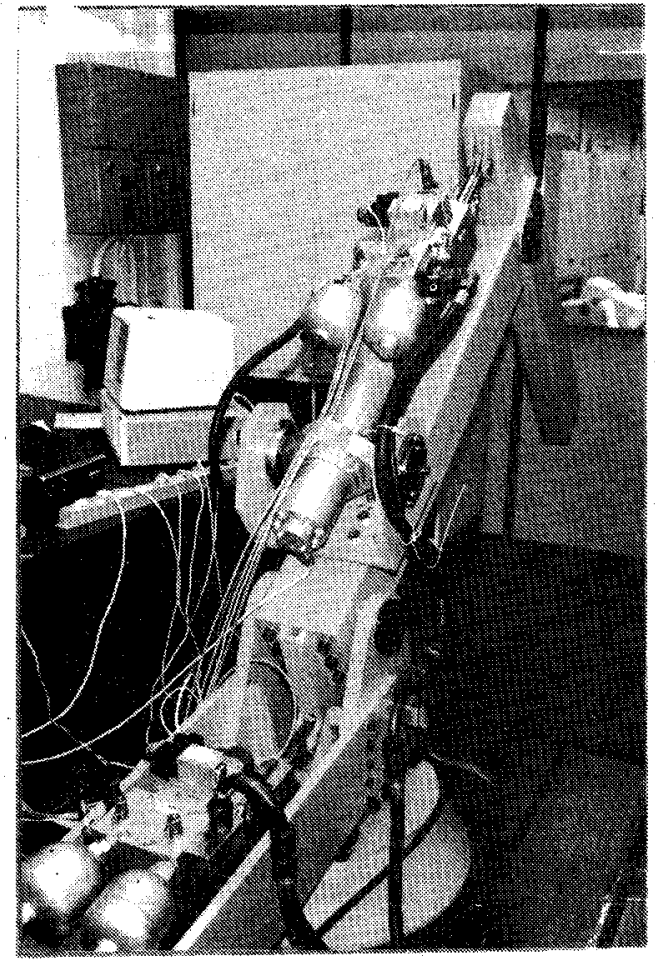

Figure 1 The constructed hydraulic test robot

force equation. Since the two actuators, one for each link, are equal, it is assumed that they have the same static and dynamic characteristic. For the sake of brevity, we shall drop the subscribes for the variables which can be applied on one of the two actuators without confusion in sequentia.

The dynamic response of the servovalves can approximately be described by the transfer function:

$$
\frac{x_{v}}{e_{g}}=\frac{k_{v}}{s^{2} / \omega_{s}^{2}+2 x_{s} s / \omega_{s}+1}
$$

where $x_{v}$ is the displacement of the spool valve from null, $e_{g}$ is the signal voltage input to the amplifier, $k_{v}$ is the amplifier gain, and $\omega_{s}(=190 \mathrm{~Hz})$ and $\xi_{s}(=0.63)$ are the natural frequency and damping ratio of the servovalve, respectively, which are estimated by means of the measured frequency response of the servovalve.

The static equation of the servovalve can approximately be described by the nonlinear flow equation:

$$
Q_{f}=\left\{\begin{array}{lll}
c_{v} w x_{v} \sqrt{\left(P_{s}-P_{f}\right) / \rho} & \text { for } & x_{v}>0 \\
c_{v} w x_{v} \sqrt{\left(P_{s}+P_{f}\right) / \rho} & \text { for } & x_{v}<0
\end{array}\right.
$$

where

Qf flow across the piston, discharge coefficient of the valve orifices. 
w area gradient of the spool valve,

$P_{\text {s }}$ supply pressure,

$P_{f}$. pressure drop across the piston, and

$\rho \quad$ mass density of oil.

Assuming that the piston is centred and that the cylinder has two symmetrical volume chambers, the continuity equation of the cylinder is:

$$
Q_{f}=A_{t} \cdot \dot{X}+\frac{V_{t}}{4 \beta} \cdot \dot{P}_{f}+c_{s l} \cdot P_{f}
$$

where:

$V_{t}$ total volume of the cylinder chambers,

$C_{s i}$ total leakage coefficient of the piston,

$\beta$ effective bulk modulus of the system,

$A_{t} \quad$ end area of the piston, and

$\dot{x}$ piston velocity.

The force equations of the two pistons are:

$$
\begin{aligned}
& A_{t} \cdot P_{1 f}=m_{t} \cdot \ddot{x}_{1}+B_{t} \cdot \dot{x}_{1}+m_{t} \cdot g \cdot \sin \left(w_{1}-\gamma\right)+F_{1} \\
& A_{t} \cdot P_{2 f}=m_{t} \cdot \ddot{x}_{2}+B_{t} \cdot \ddot{x}_{2}+m_{t} \cdot g \cdot \sin \left(\theta_{1}-w_{2}+\psi\right)+F_{2}
\end{aligned}
$$

where the subscripts 1 and 2 denote link 1 and 2, respectively, $m_{t}$ is the total mass of the piston, $B_{t}$ is the viscous damping coefficient and $F_{1}$ and $F_{2}$ are the actuator drive forces.

The joint torques and the actuator drive forces are related by the following equations:

$$
\tau_{1}=\left|d_{0} \cdot \sin (\gamma)\right| \cdot F_{1} \quad \tau_{2}=\left|b_{0} \cdot \sin (\psi)\right| \cdot F_{2}
$$

where

$$
\begin{array}{r}
-\psi=\operatorname{Arctg}\left(\frac{d_{0} \cdot \sin \left(\pi+w_{1}+V_{c m 1}-v_{1}-\theta\right)}{d_{0} \cdot \cos \left(\pi+w_{1}+V_{c m 1}-v_{1}-\theta\right)+c_{0}}\right) \\
\psi=\operatorname{Arctg}\left(\frac{a_{0} \cdot \sin \left(\pi+w_{2}+V_{c m 2}+v_{2}+\alpha\right)}{a_{0} \cdot \cos \left(\pi+w_{2}+V_{c m 2}-v_{2}+\alpha\right)+b_{0}}\right)
\end{array}
$$

where the geometric parameters are defined on Fig. 2 .

\section{CONTROLLER DESIGN AND SIMULATION RESULTS}

Fig. 3 shows the adaptive control scheme to be investigated in this paper. The control scheme consists of feedforward compensation for the gross compensation and adaptive feedback control loop for correcting the deviations from the desired trajectory.

\section{FEEDFORWARD COMPENSATION DESIGN}

If a complete dynamic compensation is carried out, the calculation of the dynamic compensation not only includes the calculation of the inverse arm dynamics, but also the transformation of the joint torques to the servo drive forces. A DSP program in C for the complete compensation requires 5547 machine states, corresponding to 0.23 msec. Based on AT\&T WE DSP32. Since the signal processor also performs the operations of the adaptive feedback control loop, which in general requires more computational time because an on-line identification should be carried out, the feedforward compensation with a heavy computational burden will limit the adaptation rates. Therefore, we should, preferably, look for approaches which can reduce the on-line calculation time of the feedforward compensation.

Fig. 4 shows a signal flow diagram for computing the feedforward compensation based on the desired joint trajectory, where the load flow $Q_{f}$ in (4) has been divided into two parts: $\bar{Q}_{f}=A_{t} \dot{x}^{d}$ and $\bar{Q}_{f}=\frac{v_{t}}{4 \beta} \dot{P}_{f}+C_{s l} P_{f}$; and the first part is the flow used to displace the piston, and the second part is the flow due to the compressibility of flow and the leakage flow. The desired joint trajectory affects the feedforward compensation along two paths: a geometrical path by means of $\left(\bar{Q}_{f}\right)$ and a dynamic path by means of $\left(\bar{Q}_{f}\right)$, where the geometrical path is independent of the robot arm dynamics. If we only use the geometrical path for the gross compensation, the computational time for the feedforward compensation will be greatly reduced.
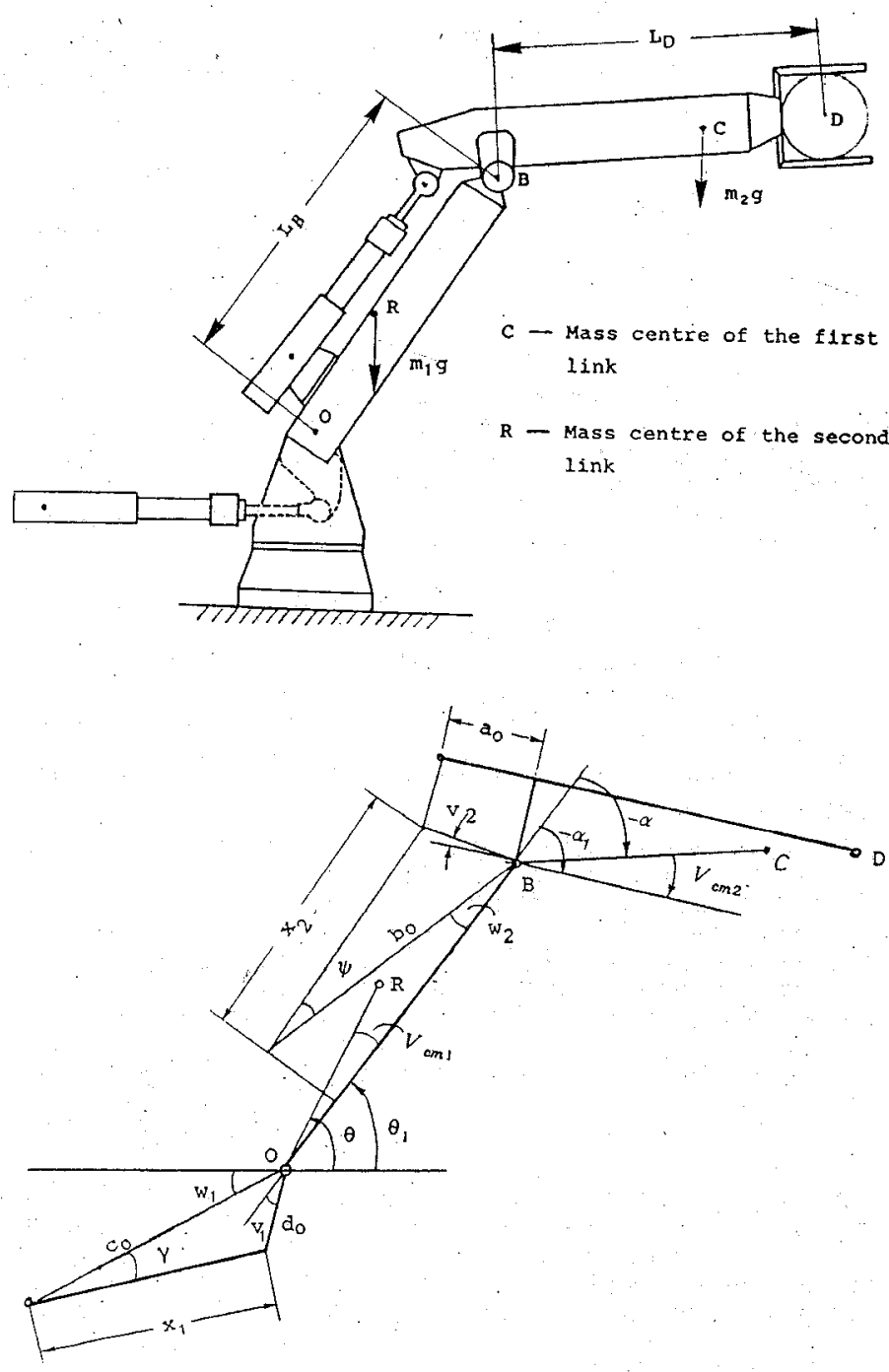

Figure 2 The link geometry of the test robot

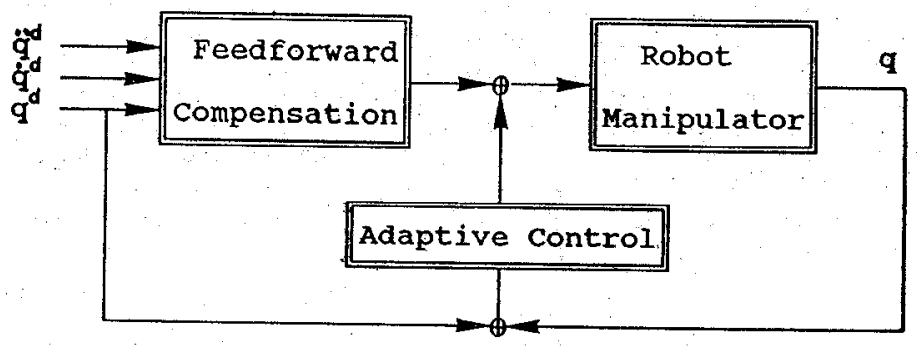

Figure 3 The adaptive control scheme with feedforward compensation 

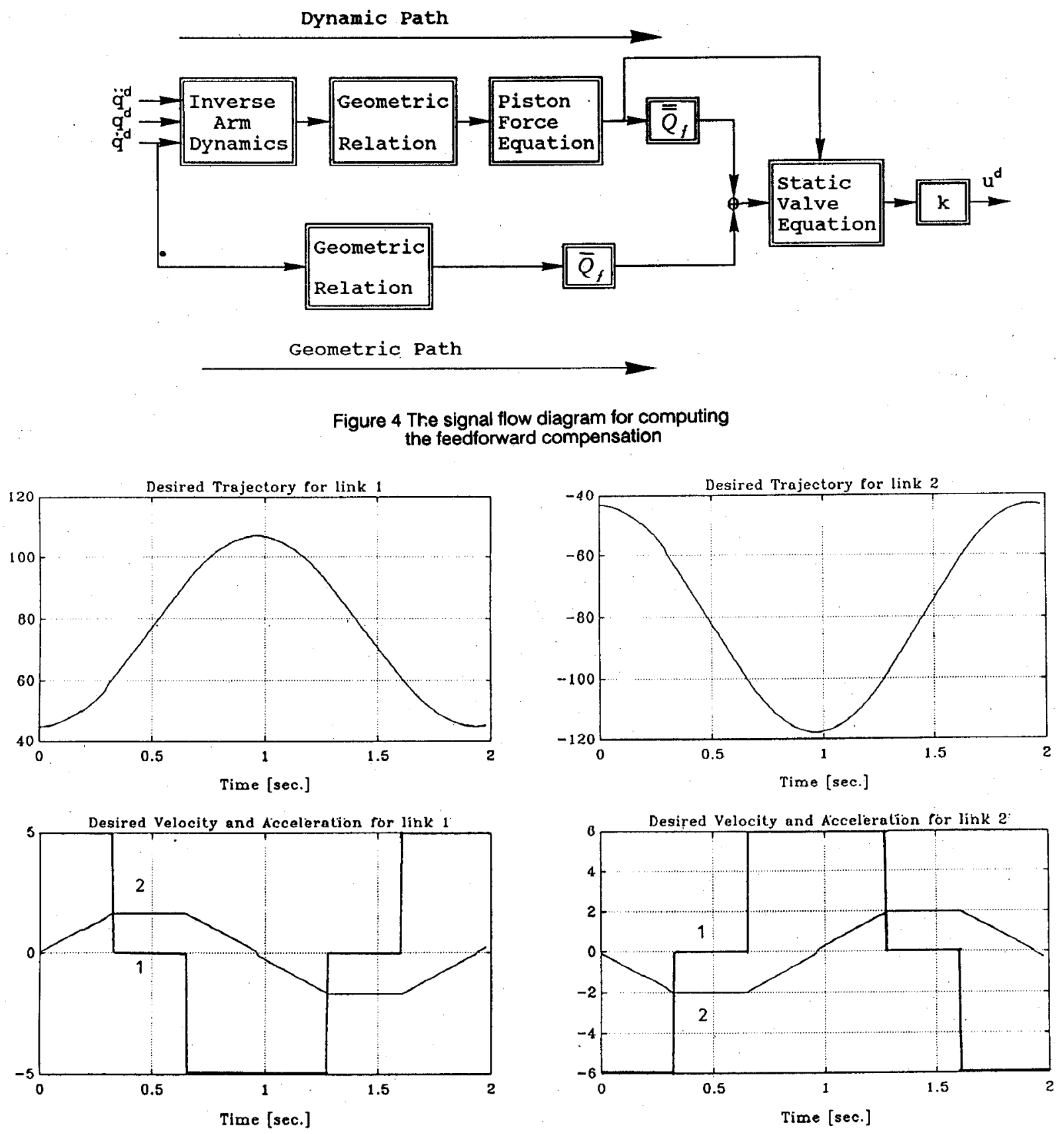

$1 \div$ Desired Acceleration
$2-$ Desired Velocity

Figure 5 The desired trajectories for the two joints

The numerical analysis and the simulation of the robot system have shown that the geometrical path plays a dominant role. For example, for the desired trajectory shown in Fig. 5, the decompositions of the continuity equations show that $Q_{f}$ has a profile similar to one that $\bar{Q}_{f}$ has, referring to Figs. 6 and 7 . For the gross compensation, furthermore, the dynamic response of the servovalve can be approximated by a constant gain $k_{g}$. As a result, the feedforward compensation can be computed by using:

$$
u^{d}(t)=\left[\begin{array}{c}
u_{1}^{d} \\
u_{2}^{d}
\end{array}\right]=\left[\begin{array}{l}
k_{1 g} \cdot A_{t} \cdot \dot{x}_{1}^{d}(t) \\
k_{2 g} \cdot A_{t} \cdot \dot{x}_{2}^{d}(t)
\end{array}\right]
$$

where $\dot{x}_{1}^{d}$ and $\dot{x}_{2}^{d}$ are the piston velocities, which are related to the desired joint velocities by the linear equations, respectively. This scheme, termed the geometrical compensation scheme, only requires three multiplications. Thus, all calculations of the inverse arm dynamics and the transformations from the joint torques to the piston drive forces are ignored. 

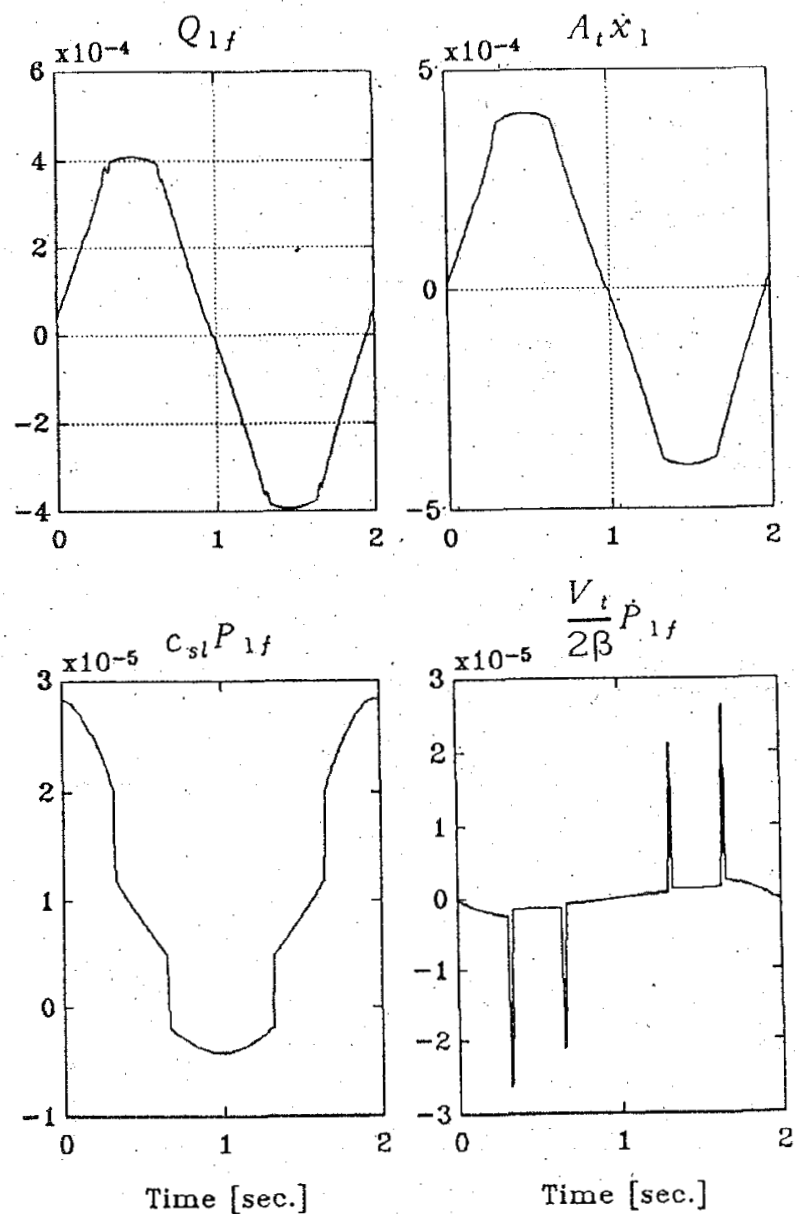

Time [sec.]

$$
Q_{1 f}=A_{i} \dot{X}_{1}+\frac{V t}{4 \beta} \dot{P}_{1 f}+c_{s l} P_{1 f}
$$

Figure 6 The decompositions of the continuity equation for the actuator 1

\section{ADAPTIVE FEEDBACK CONTROL DESIGN}

We assume that the perturbation of each joint can be modelled separately by the model:

$$
\begin{aligned}
A\left(z^{-1}\right) \delta q(k) & =B\left(z^{-1}\right) \delta u(k-1)+\frac{1}{D\left(z^{-1}\right)} e(k) \\
A\left(z^{-1}\right) & =1+a_{1} z^{-1}+a_{2} z^{-2} \\
B\left(z^{-1}\right) & =b_{0}+b_{1} z^{-1} \\
D\left(z^{-1}\right) & =1+d_{1} z^{-1}+d_{2} z^{-2} \\
\delta q(k) & =q^{\alpha}(k)-q(k)
\end{aligned}
$$

where $k$ denotes the sampling instant. Based on the linear model, the generalized minimum variance controller [5-6] can be used to compute the feedback control. The cost function is

$$
J=E\left\{(\delta q(k+1))^{2}+\mu(\delta u(k))^{2}\right\}
$$

where $E(\cdot)$ is the expectation operator conditional on data up to time $k$; and $\mu$ is the control weight. The minimization of the cost function gives:

$$
\delta u(k)=-\frac{G \delta q(k)}{\hat{D} \hat{B}+\mu / \hat{\sigma}_{0}}
$$
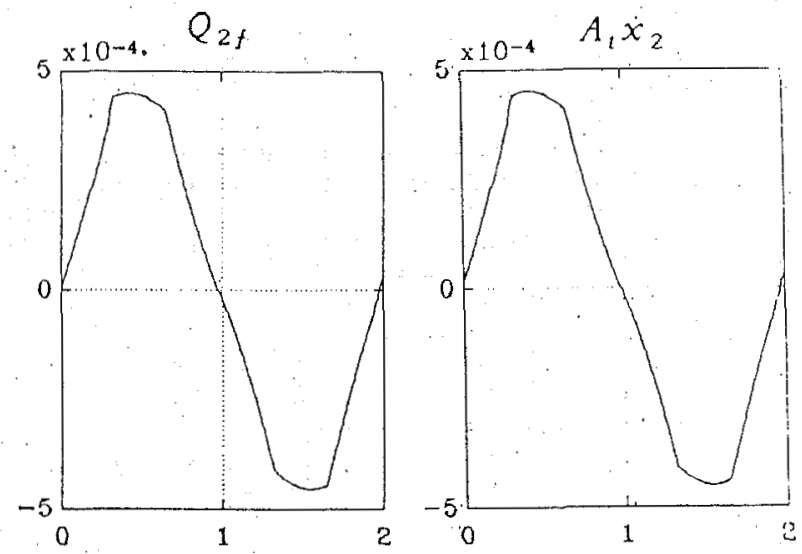

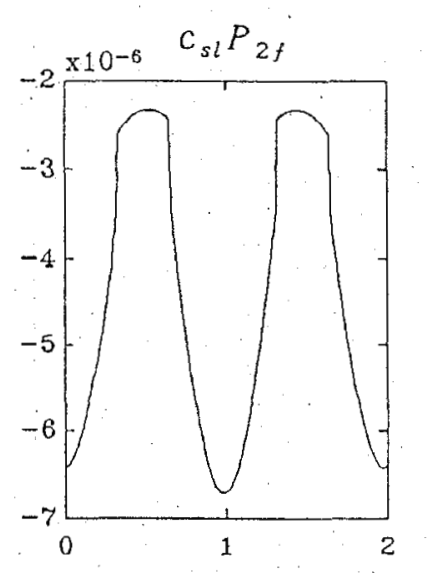

Time [sec.]

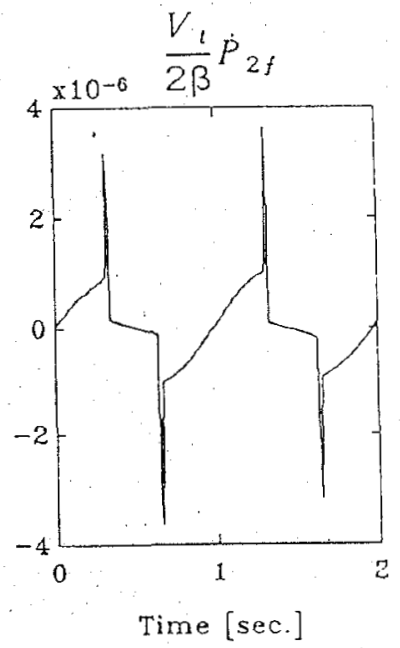

$$
Q_{2 f}=A_{1} \dot{x}_{2}+\frac{1}{H \beta} \dot{p}_{2 f}+c_{s l} P_{\lambda l}
$$

Figure 7 The decompositions of the continuity equation for the actuator 2

$$
G=z(1-\hat{A} \bar{D})
$$

where "?" denotes the estimates of the unknown polynomials $i$, is and $D$ by using the recursive generalized least squares algorithm.

\section{SIMULATION RESULTS}

Our simulations show that for a robot manipulator in which the mass centre of the link is not close to the joint, the geometrical scheme might give big trajectory deviations when the links move from the high position to the low position at high speed. The reason for that is that the amplitude of the compensation control is too big in that period due to the lack of the robot arm gravity compensation. This is confirmed by a comparison of the complete (dynamic and geometric) compensation and the geometric compensation, shown in Fig. 8 based on the trajectory shown in Fig. 5. A remedy to reduce the effect of gravity is to multiply the compensation control by a gravity compensation factor in the period when the link moves from the high position to the low position.

Fig. 9 presents the trajectory trackings and control inputs of the two links for the modified geometrical compensation scheme, where the compensation control is multiplied by 0.7 in the last half part of the trajectory for the link 1 and by 0.8 in the first half part of the trajectory for the link 2. The maximum position errors are $2.53^{\circ}$ for the joint 1 and $3.25^{\circ}$ for the joint 2 . The trajectory tracking performance of the two links is close to the results obtained with the complete dynamics compensation scheme. 


\section{CONCLUSIONS}

An adaptive geometric compensation scheme for hydraulic manipulators have been proposed and investigated. The adaptive geometrical compensation scheme has the advantages of less computation time and simplicity. Another important advantage is that it does not require any knowledge of the dynamic model of the robot a'm. The feasibility study of implementing the regarded adaptive control scheme proposed has shown that it is possible to implement the adaptive control scheme on a fast robot manipulator based on available processors: For example, a DSP program in C for implementing the regarded control scheme requires total 36900 machine states, corresponding to $1.48 \mathrm{msec}$. Based on the AT\&T WE DSP32 Digital Signal Processor. Experimental study of implementing the control schemes on the hydraulic test robot is now being carried out at our Institute.

\section{REFERENCES}

[1] Paul, R. C., "Modeling, Trajectory Calculation and Servoing of a Computed Control Arm," Rep. AlM-177; Stanford Univ. Artificial Intelligence Lab., Sept., 1972.

[2] Liegeois, A., A. Fourmier, and M. Aldon, "Model Reference Control of High-Velocity Industrial Robots," Proc. Joint Automatic Control Conf., San Fransiso, CA, Aug. 13-15, 1980:

[3] Lee, C. S. G. and M. J. Chung, "An Adaptive Control Strategy for Mechanical Manipulators", IEEE Trans., on Auto. Control. Vol AC-29, 1984, No. 9, pp. 837-840.

[4] Conrad, F., P. H. Sørensen, E. Trostmann, J. J. Zhou, "Design and Digital Control of a Fast Hydraulic Test Robot Manipulator," Proc. of the 9th BHRA International Conference On Fluid Power, Cambridge, England, 25-27 April, 1990.

[5] Zhou Jian-jun, Adaptive Control and Applications to Hydraulic Robot Manipulators. Ph.D. Thesis, the Control Engineering Institute, Technical University of Denmark, 1989.

[6] Clarke, D. W. and P. J. Gawthrop, "Self-Tuning Controller", IEE Proc., 122. Pt. D, No. 9, pp. 929-934, 1975.
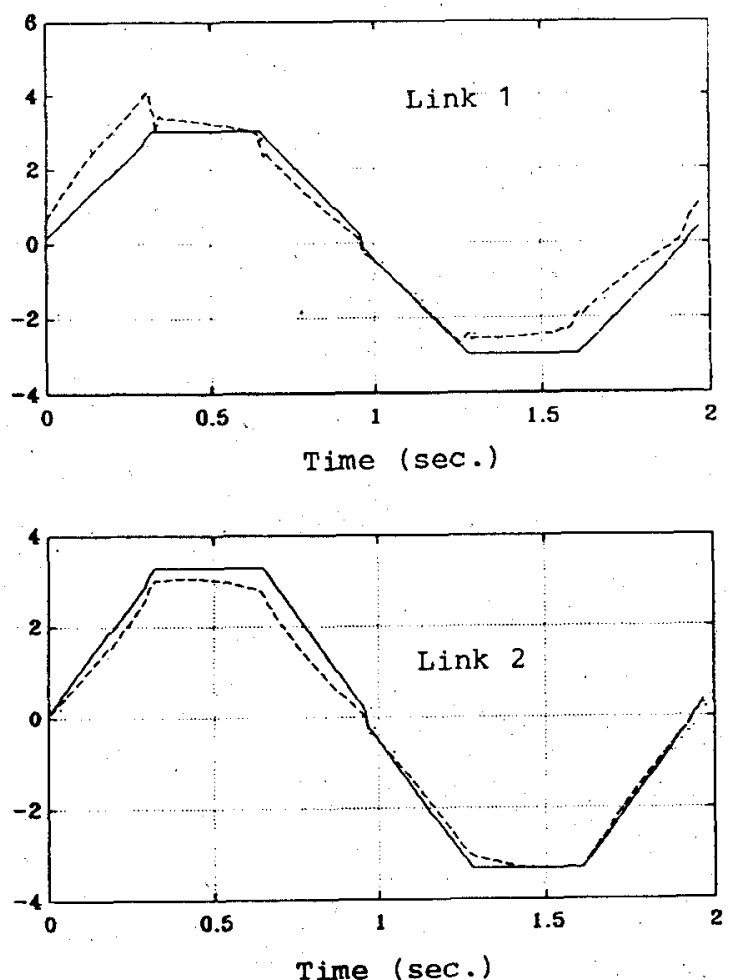

\section{--- Complete compensation Geometric compensation}

Figure 9 The simulation results
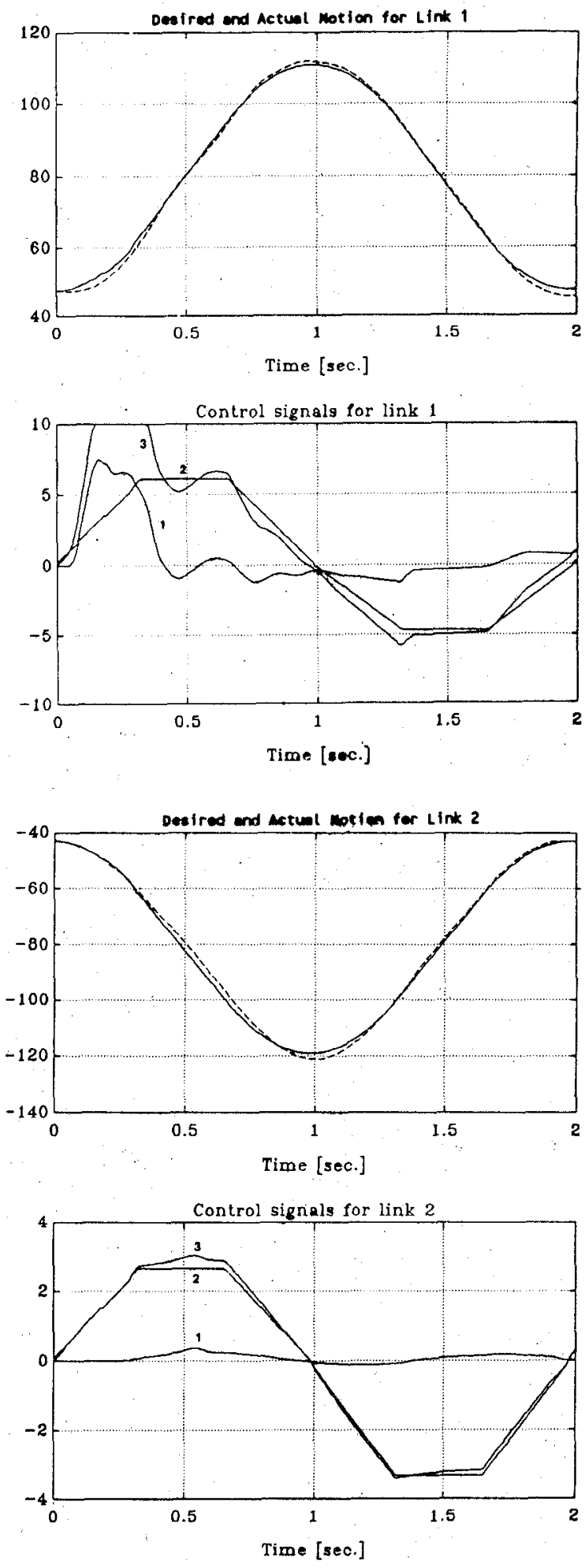

1 - Adaptive Control, -- Actual Trajectory
2 Nominal Control, $3 \longrightarrow$ Actual Control

Figure 8 Comparison of the complete compensation and the geometric compensation 